\title{
ARTICLE
}

\section{Kinetic Monte Carlo Simulations of Initial Process of Solute Atom Cluster Formations Based on ab initio Data Base}

\author{
Kiyoshi BETSUYAKU*, Toshiharu OHNUMA and Naoki SONEDA \\ Material Science Research Laboratory, Central Research Institute of Electric Power Industry, \\ 2-11-1 Iwado Kita, Komae-shi, Tokyo 201-8511, Japan
}

\begin{abstract}
An accurate prediction of embrittlement of neutron-irradiated reactor pressure vessel steel is very important for the long term operation of light water reactors. Although solute atom clusters formed in the steel have been observed by using three dimensional atom probe technique, understanding of the elementary mechanisms of the formation of the clusters have not been established. We have carried out $a b$ initio calculations to determine the interactions among solvent, solute atoms and vacancies, and migration barriers in Fe based dilute alloys and built a database used to parameterize pair energies for kinetic lattice Monte Carlo simulations. The result of calculation shows surplus vacancies act as nucleus of small $\mathrm{Cu}$ clusters and are trapped by the clusters, and the number of clusters depends on the number of vacancies in the system. This indicates the importance of vacancy concentrations in the alloys caused by irradiations for the initial process of the solute atom cluster formation.
\end{abstract}

KEYWORDS: reactor pressure vessel steel, irradiation embrittlement, solute atom cluster, kinetic lattice Monte Carlo calculation, ab initio calculation

\section{Introduction}

Reactor pressure vessel (RPV) steels contain additive elements and impurities such as $\mathrm{Cu}, \mathrm{Ni}, \mathrm{Mn}$, and $\mathrm{Si}$ besides Fe. These solute atoms are accumulated by the influence of the neutron irradiation and form solute atom clusters. ${ }^{1,2)}$ The solute atom clusters obstruct the movements of dislocations and cause embrittlement of the RPV steel. The clusters which formed mainly from $\mathrm{Cu}$ precipitations have been assumed to be one of main causes of embrittlement. However, there are still a lot of uncertainties of the process of the cluster formations (e.g. contribution of other solute atoms such as $\mathrm{Ni}, \mathrm{Mn}$, and $\mathrm{Si}$ ). The results of measurements that cannot be explained by precipitations of $\mathrm{Cu}$ (such as dilute $\mathrm{Cu}$ clusters or clusters enriched with the other solute atoms) are obtained by threedimensional atom probe (3DAP) for the low $\mathrm{Cu}$ steel alloys. ${ }^{2)}$

While the experimental studies such as the microstructure observations by 3DAP and transmission electron microscopy have progressed, the quantitative computer simulation technique that can be compared directly with the experimental data is needed in the theoretical study.

In the present study, early process of the formation of solute atom clusters is investigated by combining the $a b$ initio calculation with kinetic lattice Monte Carlo (KLMC) method., ${ }^{3,4)}$

\section{Calculation Methods}

\section{Ab initio Calculations}

$A b$ initio calculations based on the density functional theory have been applied over the wide range of the materials

*Corresponding author, E-mail: betuyaku@criepi.denken.or.jp

(C) 2011 Atomic Energy Society of Japan, All Rights Reserved. science, and are effective to discuss the structural stability of the materials, especially. The calculations have been applied to irradiation embrittlement problems and have succeeded to explain the state of defects in RPV steels. ${ }^{5-7)}$ The interaction energies shown as follows are obtained from total energy calculations.

The binding energy of two kinds of solute atoms $X$ and $Y$ $E_{X Y}^{b}$ is given by

$$
\begin{aligned}
E_{X Y}^{b}= & E[(N-1) \mathrm{Fe}+X]+E[(N-1) \mathrm{Fe}+Y] \\
& -E[(N-2) \mathrm{Fe}+X+Y]-E[N \mathrm{Fe}],
\end{aligned}
$$

where $E[(N-1) \mathrm{Fe}+X], E[(N-1) \mathrm{Fe}+Y]$ and $E[(N-$ $2) \mathrm{Fe}+X+Y]$ are the total energy for the supercell with impurities $X, Y$ and both $X$ and $Y$, respectively.

The migration energy $E^{m i g}$ is assumed to be a total energy difference between for atom being at saddle point $E^{S P}$ and for initial stable point $E^{i n i}$ :

$$
E^{m i g}=E^{S P}-E^{i n i} .
$$

The vacancy formation energy $E_{V a}^{f o r}$ in Fe is calculated by the following expression:

$$
E_{V a}^{f o r}=E[(N-1) \mathrm{Fe}+V a]-(N-1) E[\mathrm{Fe}],
$$

where $V a$ expresses vacancy, $E[(N-1) \mathrm{Fe}+V a]$ is the total energy for supercell containing $(N-1) \mathrm{Fe}$ atoms and a vacancy and $E[\mathrm{Fe}]=E[N \mathrm{Fe}] / N$ is the total energy for bulk $\mathrm{Fe}$.

The solution energy for a solute atom $X$ is given by follows:

$$
E_{X}^{s o l}=E[(N-1) \mathrm{Fe}+X]-(N-1) E[\mathrm{Fe}]-E_{r e f}[X],
$$


where $E_{r e f}[X]$ is a reference energy for the solute atom.

\section{Kinetic Lattice Monte Carlo Calculations}

The KLMC method using in the present study is based on the rigid lattice model with pair interactions. ${ }^{8)}$ In the method, $\mathrm{Fe}$ atoms, solute atoms, and vacancies move on the fixed lattice point. The atoms move along with the jump of vacancies to the neighbor sites. As a result, the process of diffusion and precipitation of atoms is simulated.

The probability of vacancy jump to $X$ atom site $\Gamma_{X}$ is given by follows:

$$
\Gamma_{X}=\nu_{X} \exp \left(-\frac{E_{a}}{k_{\mathrm{B}} T}\right),
$$

where $k_{\mathrm{B}}$ is Boltzmann constant, $T$ is a temperature, $\nu_{X}$ is a specific frequency and $E_{a}$ is an activation energy.

The total energy of the system is given by the sum of the pair interactions of the components (atoms and vacancies):

$$
E=\frac{1}{2} \sum_{i} \sum_{X, Y} \varepsilon_{X Y}^{(i)},
$$

where $\varepsilon_{X Y}^{(i)}$ is pair interaction for elements $X$ and $Y$ on the $i$-th nearest neighbors. The effects of lattice distortions near solute atoms and vacancies are included in the pair interactions.

A time step $\tau$ is given by follows:

$$
\tau=-\log \xi / \Pi
$$

where $\Pi=\sum \Gamma$ is total sum of the jump probabilities and $\xi$ is a random number on interval $(0,1] .{ }^{9)}$

Although the activation energy depends on the atomic configurations around vacancies, it is difficult to calculate the energy for each of the configurations. In order to consider the effect of atomic configuration change, we use the activation energy defined by follows: ${ }^{4)}$

$$
E_{a}=E^{m i g}+\frac{E_{f}-E_{i}}{2},
$$

where $E_{i}$ is the total energy of the system before vacancy jump and $E_{f}$ is of after the jump. $E^{m i g}$ is migration energy depending only on kind of atom.

The cohesive energy using pair interactions between same kind of atom on $i$-th nearest neighbor site is given by follows:

$$
E_{b c c}^{c o h}[X]=\sum_{i} \frac{z_{i}}{2} \varepsilon_{X X}^{(i)},
$$

where $z_{i}$ is the number of $i$-th nearest neighbor atoms. $X$ indicates Fe or solute atom and $E_{b c c}^{c o h}[X]$ means cohesive energy for a bcc $X$ crystal.

The vacancy formation energy using pair interaction between $\mathrm{Fe}$ and vacancy is given by follows:

$$
E_{V a}^{f o r}=\sum_{i}\left(-\frac{z_{i}}{2} \varepsilon_{\mathrm{FeFe}}^{(i)}+z_{i} \varepsilon_{\mathrm{Fe} V a}^{(i)}\right) .
$$

The binding energy using pair interactions between Fe and solute atoms, and between vacancy and solute atoms is given by follows:

$$
E_{X Y}^{b(i)}=\varepsilon_{\mathrm{Fe} X}^{(i)}+\varepsilon_{\mathrm{Fe} Y}^{(i)}-\varepsilon_{\mathrm{FeFe}}^{(i)}-\varepsilon_{X Y}^{(i)},
$$

where $X$ and $Y$ indicate solute atoms or vacancy.

The solution energy of a solute atom $X$ for Fe using pair interactions between $\mathrm{Fe}$ and $X$ is given by follows:

$$
E_{X}^{s o l}=-\sum_{i} \frac{z_{i}}{2}\left(\varepsilon_{\mathrm{FeFe}}^{(i)}+\varepsilon_{X X}^{(i)}-2 \varepsilon_{\mathrm{Fe} X}^{(i)}\right) .
$$

\section{Results}

\section{Ab initio Calculations}

$A b$ initio calculations have been performed by using VASP code. ${ }^{10,11)}$ The generalized gradient approximation (GGA) was applied for exchange and correlation potentials and the GGA parameterization described by Perdew et al. was used. ${ }^{12)}$ We used projector augmented-wave (PAW) method $^{13,14)}$ for electronic structure calculations. Cutoff energy for plane waves was set to $400 \mathrm{eV} .4 \times 4 \times 4$ supercells containing 128 atoms were used. k point sampling in the Brillouin zone was $3 \times 3 \times 3$ Monkhorst-Pack mesh. The effects of spin polarization were included. The defect calculations were performed with full relaxation.

Calculation results of binding energies, migration energies and vacancy formation energy are collected in Table 1 These results show following characteristics. For $\mathrm{Cu}$, there are strong attractive interactions between 1 st nearest $\mathrm{Cu}-\mathrm{Cu}$ pair and both between 1 st and 2 nd nearest $\mathrm{Cu}-\mathrm{Va}$ pairs. These cause precipitation of $\mathrm{Cu}$ without dissociation of $\mathrm{Cu}-\mathrm{Va}$ pairs in $\mathrm{Fe}-\mathrm{Cu}$ alloy. ${ }^{15)}$ For $\mathrm{Si}$, there are repulsive interactions both between 1 st and 2 nd nearest $\mathrm{Si}$-Si pair, and attractive interaction between 1 st nearest $\mathrm{Si}-\mathrm{Va}$ pair. For $\mathrm{Ni}$, there are small interactions between $\mathrm{Ni}-\mathrm{Ni}$ pairs and strong attractive interaction between 2 nd nearest $\mathrm{Ni}-\mathrm{Va}$ pair.

\section{Kinetic Lattice Monte Carlo Calculations}

From ab initio calculation data, we determined the pair interaction energies for KLMC calculation. We considered pair interaction up to 2 nd nearest neighbor.

The pair interaction energies collected in Table $\mathbf{2}$ are determined by following way.

From Eq. (9), pair energies between same kind of atom $\varepsilon_{X X}^{(i)}(X=\mathrm{Fe}, \mathrm{Cu}, \mathrm{Ni}$ or $\mathrm{Si})$ are determined as

$$
\begin{gathered}
\varepsilon_{X X}^{(1)}=-E_{b c c}^{c o h}[X] /\left(\frac{z_{1}}{2}+\frac{z_{2}}{2} \alpha\right), \\
\varepsilon_{X X}^{(2)}=\alpha \varepsilon_{X X}^{(1)},
\end{gathered}
$$

where $\alpha=1 / 2$ is adopted the same as Soisson and $\mathrm{Fu}^{3)} \mathrm{Co}$ hesive energies listed in Table $\mathbf{3}$ are based on experimental values $^{16)}$ because $a b$ initio calculation is known not to give reliable cohesive energies. Since stable crystal structures for $\mathrm{Cu}, \mathrm{Ni}$ and $\mathrm{Si}$ are not bcc, we calculated energy difference between bcc and the stable structure.

$\varepsilon_{\mathrm{FeVa}}^{(i)}$ is determined from the vacancy formation energy relation (10):

$$
\begin{gathered}
\varepsilon_{\mathrm{Fe} V a}^{(1)}=\left(E_{V a}^{\text {for }}+\frac{z_{1}}{2} \varepsilon_{\mathrm{FeFe}}^{(1)}+\frac{z_{2}}{2} \varepsilon_{\mathrm{FeFe}}^{(2)}\right) /\left(z_{1}+z_{2} \beta\right), \\
\varepsilon_{\mathrm{Fe} V a}^{(2)}=\beta \varepsilon_{\mathrm{Fe} V a}^{(1)},
\end{gathered}
$$

where $\beta=1 / 2$ is adopted. ${ }^{3)}$ 
Table 1 Binding energies for 1st and 2nd nearest neighbor, migration energies and vacancy formation energy in Fe obtained from $a b$ initio calculations.

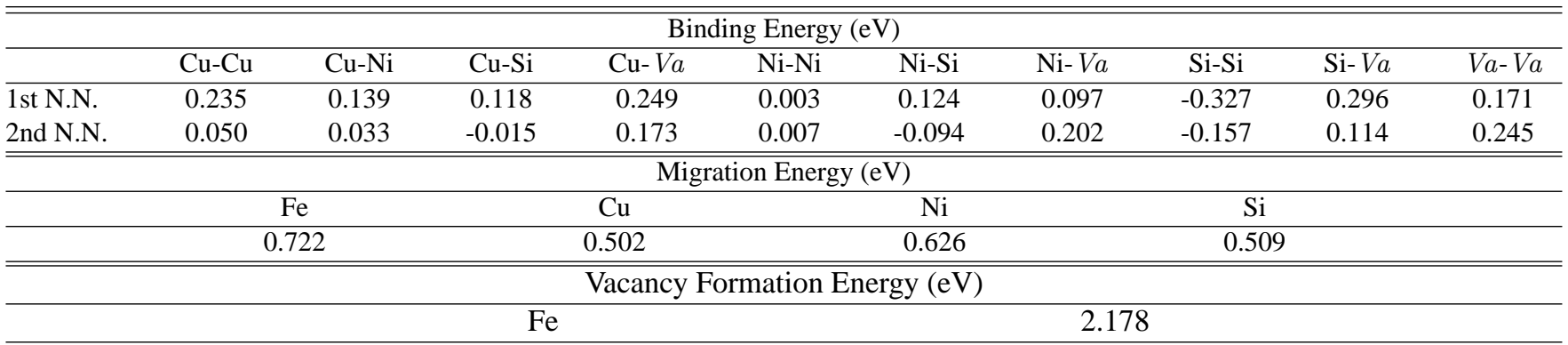

Table 2 1st and 2nd nearest neighbor pair interaction energies.

\begin{tabular}{ccc}
\hline \hline Pair energy $(\mathrm{eV})$ & $1 \mathrm{st}(i=1)$ & 2nd $(i=2)$ \\
\hline$\varepsilon_{\mathrm{FeFe}}^{(i)}$ & -0.778 & -0.389 \\
$\varepsilon_{\mathrm{FeCu}}^{(i)}$ & -0.585 & -0.326 \\
$\varepsilon_{\mathrm{FeNi}}^{(i)}$ & -0.783 & -0.389 \\
$\varepsilon_{\mathrm{FeSi}}^{(i)}$ & -0.926 & -0.460 \\
$\varepsilon_{\mathrm{FeVa}}^{(i)}$ & -0.191 & -0.096 \\
$\varepsilon_{\mathrm{CuCu}}^{(i)}$ & -0.627 & -0.314 \\
$\varepsilon_{\mathrm{CuNi}}^{(i)}$ & -0.729 & -0.359 \\
$\varepsilon_{\mathrm{CuSi}}^{(i)}$ & -0.851 & -0.382 \\
$\varepsilon_{\mathrm{Cu} V a}^{(i)}$ & -0.247 & -0.206 \\
$\varepsilon_{\mathrm{NiNi}}^{(i)}$ & -0.791 & -0.395 \\
$\varepsilon_{\mathrm{NiSi}}^{(i)}$ & -1.055 & -0.366 \\
$\varepsilon_{\mathrm{Ni} V a}^{(i)}$ & -0.293 & -0.297 \\
$\varepsilon_{\mathrm{SiSi}}^{(i)}$ & -0.747 & -0.374 \\
$\varepsilon_{\mathrm{Si} \text { Va }}^{(i)}$ & -0.635 & -0.280 \\
$\varepsilon_{V a}^{(i)}$ & 0.225 & -0.047 \\
\hline
\end{tabular}

From binding energy between vacancies $E_{V a V a}^{b(i)}$ and Eq. (11), $\varepsilon_{V a V a}^{(i)}$ is determined as

$$
\varepsilon_{V a V a}^{(i)}=-E_{V a V a}^{b(i)}+2 \varepsilon_{\mathrm{Fe} V a}^{(i)}-\varepsilon_{\mathrm{FeFe}}^{(i)}, \quad i=1,2 .
$$

Pair interactions between $\mathrm{Fe}$ and solute atoms $\varepsilon_{\mathrm{Fe} X}^{(i)}(X=$ $\mathrm{Cu}, \mathrm{Ni}$ or $\mathrm{Si}$ ) are determined from binding energies between same kind of atom $E_{X X}^{b(i)}$ and Eq. (11):

$$
\varepsilon_{\mathrm{Fe} X}^{(i)}=\left(E_{X X}^{b(i)}+\varepsilon_{\mathrm{FeFe}}^{(i)}+\varepsilon_{X X}^{(i)}\right) / 2, \quad i=1,2 .
$$

Pair interactions between solute atoms and vacancy $\varepsilon_{X V a}^{(i)}(X=\mathrm{Cu}, \mathrm{Ni}$ or $\mathrm{Si})$ are determined from binding energies between solute atoms and vacancy $E_{X V a}^{b(i)}$ and Eq. (11):

$$
\varepsilon_{X V a}^{(i)}=-E_{X V a}^{b(i)}+\varepsilon_{\mathrm{Fe} V a}^{(i)}+\varepsilon_{\mathrm{FeX}}^{(i)}-\varepsilon_{\mathrm{FeFe}}^{(i)}, \quad i=1,2 .
$$

From binding energies between different kind of solute atoms $E_{X Y}^{b(i)}(X, Y=\mathrm{Cu}, \mathrm{Ni}$ or Si, $X \neq Y)$ and Eq. (11), we
Table 3 Cohesive energies used to determine pair interactions between same kind of atom.

\begin{tabular}{cc}
\hline \hline & Cohesive energy $(\mathrm{eV})$ \\
\hline $\mathrm{Fe}$ & 4.28 \\
$\mathrm{Cu}$ & 3.45 \\
$\mathrm{Ni}$ & 4.35 \\
$\mathrm{Si}$ & 4.11 \\
\hline
\end{tabular}

Table 4 Comparison between solution energies by VASP and those obtained from pair interactions (PI).

\begin{tabular}{lcc}
\hline \hline Solution energy (eV) & VASP & PI \\
\hline $\mathrm{Cu}$ & 0.696 & 1.090 \\
$\mathrm{Ni}$ & 0.003 & 0.033 \\
$\mathrm{Si}$ & -1.690 & -1.779 \\
\hline
\end{tabular}

determine $\varepsilon_{X Y}^{(i)}$ as follows:

$$
\varepsilon_{X Y}^{(i)}=-E_{X Y}^{b(i)}+\varepsilon_{\mathrm{Fe} X}^{(i)}+\varepsilon_{\mathrm{Fe} Y}^{(i)}-\varepsilon_{\mathrm{FeFe}}^{(i)}, \quad i=1,2 .
$$

We did not use ab initio solution energies for determinations of pair interaction because the energies have ambiguities for their reference state. Table $\mathbf{4}$ shows a comparison between solution energies by VASP and those obtained from pair interactions. The reference state for solute atom is set to bulk bcc structure. Although the resulting solution energies are somewhat different, the solubility trend for each solute atom is consistent because the energies agree in signs and magnitude relations. Therefore these differences might not cause significant difference in simulation results.

We adopted Debye frequencies reduced from Debye temperature $^{16)}$ as attempt frequencies (Table 5 ).

KLMC calculations were performed on $\mathrm{Fe}-\mathrm{Cu}-\mathrm{Ni}-\mathrm{Si}$ alloy. The compositions were set to $\mathrm{Cu}: 0.27 \mathrm{at} . \%, \mathrm{Ni}: 1.72 \mathrm{at} . \%$ and $\mathrm{Si}: 0.40$ at.\% being close to the high $\mathrm{Cu}$ model alloy including $\mathrm{Cu}, \mathrm{Ni}, \mathrm{Mn}$ and $\mathrm{Si}$. The system size was 32768 $(=32 \times 32 \times 32)$ lattice with periodic boundary condition. Simulation temperature was set to $800 \mathrm{~K}$ which is higher than that of RPV operation $(\sim 560 \mathrm{~K})$. Calculated migration energy for Fe is higher than the experimental value $\left(0.55 \mathrm{eV}^{17)}\right)$; the tendency is similar to previous calculations $(0.67 \mathrm{eV}$ and 
Table 5 Attempt frequencies used in the calculations (Debye frequency).

\begin{tabular}{cc}
\hline \hline & Attempt frequency $\left(\mathrm{s}^{-1}\right)$ \\
\hline $\mathrm{Fe}$ & $9.79 \times 10^{12}$ \\
$\mathrm{Cu}$ & $7.16 \times 10^{12}$ \\
$\mathrm{Ni}$ & $9.38 \times 10^{12}$ \\
$\mathrm{Si}$ & $1.34 \times 10^{13}$ \\
\hline
\end{tabular}

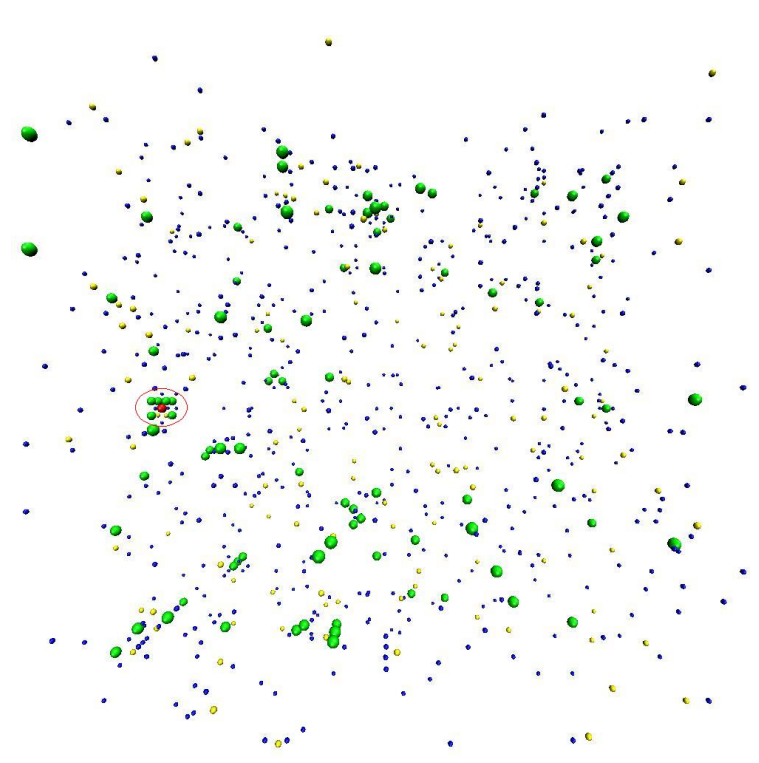

Fig. 1 Spatial distribution of atoms for Fe-Cu-Ni-Si alloy obtained from KLMC calculation with 1 vacancy $9.35 \mathrm{~s}$ after the initial condition. (001) plane is parallel to the sheet. Green, blue, yellow, and red balls indicate $\mathrm{Cu}, \mathrm{Ni}, \mathrm{Si}$, and vacancy, respectively. Solute atom cluster with vacancy is encircled by red circle.

$0.65 \mathrm{eV}) .^{3,5)}$ Therefore we used the temperature in order to accelerate simulation progress. Random distributions of solute atoms and vacancies were used as initial conditions.

Figure 1 shows the result with 1 vacancy in the system 9.35 $\mathrm{s}$ after the initial condition. The vacancy acts as a nuclear of $\mathrm{Cu}$ cluster and is trapped by the cluster. Figure 2 shows the result with 5 vacancies in the system $2.25 \mathrm{~s}$ after the initial condition. $\mathrm{Cu}$ clusters are also formed near vacancies.

In order to examine the dependence of the number of clusters on the number of vacancies, we carried on calculations with increasing them up to 9 . Figure 3 shows the dependence of the number of clusters on the number of vacancies $1.7 \mathrm{~s}$ after the initial condition. The number of clusters increases as the number of vacancies increases. The situations correspond to surplus vacancies caused by irradiation and the results indicate the importance of vacancy concentrations in the alloys for the initial process of the formation of solute atom clusters.

\section{Conclusion}

We have performed $a b$ initio calculations and KLMC calculations. From the results of $a b$ initio calculations, we determined the pair interaction energy for KLMC calculations.

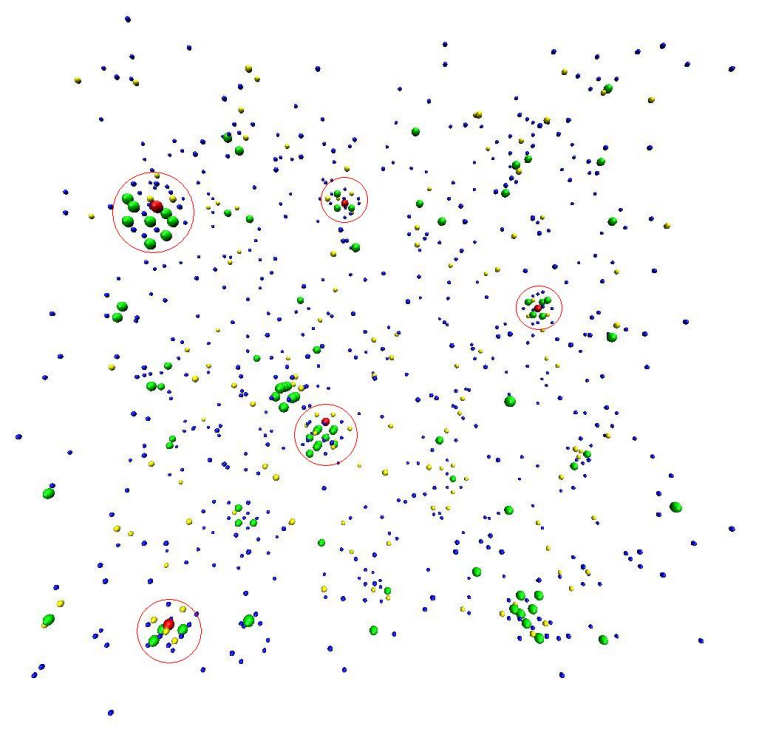

Fig. 2 Spatial distribution of atoms for $\mathrm{Fe}-\mathrm{Cu}-\mathrm{Ni}-\mathrm{Si}$ alloy obtained from KLMC calculation with 5 vacancies $2.25 \mathrm{~s}$ after the initial condition. (001) plane is parallel to the sheet. Green, blue, yellow, and red balls indicate $\mathrm{Cu}, \mathrm{Ni}, \mathrm{Si}$, and vacancy, respectively. Solute atom clusters with vacancies are encircled by red circles.

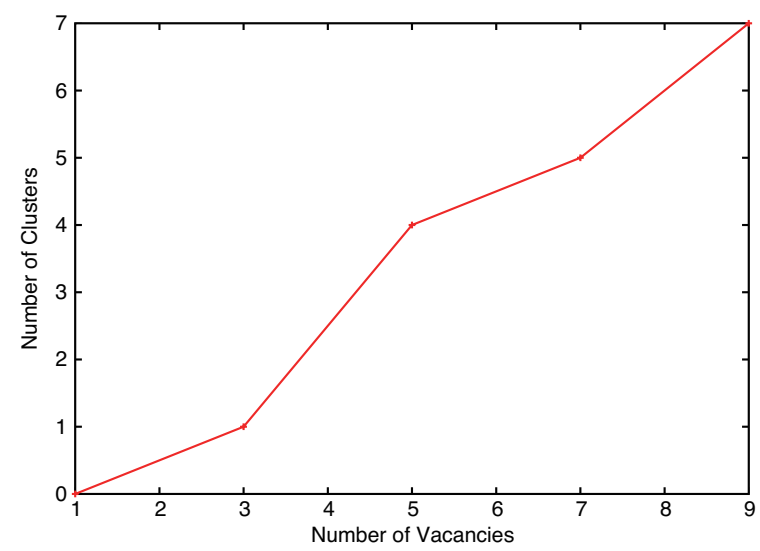

Fig. 3 The dependence of the number of clusters on the number of vacancies $1.7 \mathrm{~s}$ after the initial condition.

The results of KLMC calculations with plural vacancies show each of the vacancies acts as the nuclear of $\mathrm{Cu}$ cluster and the number of clusters increases as the number of vacancies increases. These indicate the importance of surplus vacancies caused by irradiation for the initial process of the formation of solute atom clusters.

In the present work, we neglected effects of self interstitial atom (SIA) which assumes to play an important role under irradiation environments. SIA clusters and SIA loops formed by irradiations are also supposed to contribute to embrittlement. Therefore combining the behavior of vacancies and SIAs, it will be possible to perform more quantitative analysis and to make more accurate prediction of embrittlement. The simulations including SIAs are in progress. 


\section{References}

1) R. G. Carter, N. Soneda, K. Dohi, J. M. Hyde, C. A. English, W. L. Server, "Microstructural characterization of irradiationinduced Cu-enriched clusters in reactor pressure vessel steels," J. Nucl. Mater., 298[3], 211-224 (2001).

2) P. Auger, P. Pareige, M. Akamatsu, J.-C. Van Duysen, "Microstructural characterization of atom clusters in irradiated pressure vessel steels and model alloys," J. Nucl. Mater, 211[3], 194-201 (1994).

3) F. Soisson, C. C. Fu, "Cu-precipitation kinetics in $\alpha$-Fe from atomistic simulations: Vacancy-trapping effects and $\mathrm{Cu}$-cluster mobility," Phys. Rev., B76[21], 214102 (2007).

4) E. Vincent, C. S. Becquart, C. Pareige, P. Pareige, C. Domain, "Precipitation of $\mathrm{FeCu}$ system: A critical review of atomic kinetic Monte Carlo simulations," J. Nucl. Mater., 373[1-3], 387401 (2008).

5) C. Domain, C. S. Becquart, "Ab initio calculations of defects in Fe and dilute Fe-Cu alloys,” Phys. Rev., B65[2], 024103 (2001).

6) E. Vincent, C. S. Becquart, C. Domain, "Ab initio calculations of vacancy interactions with solute atoms in bcc Fe," Nucl. Instr. Meth. Phys. Res., B228[1-4], 137-141 (2005).

7) C. C. Fu, F. Willaime, P. Ordejon, "Stability and mobility of mono- and di-interstitials in $\alpha$-Fe," Phys. Rev. Lett., 92[17], 175503 (2004).

8) Y. L. Bouar, F. Soisson, "Kinetic pathways from embeddedatom-method potentials: Influence of the activation barriers," Phys. Rev., B65[9], 094103 (2002).
9) C. C. Battaile, D. J. Srolovitz, J. E. Butler, "A kinetic Monte Carlo method for the atomic-scale simulation of chemical vapor deposition: Application to diamond," J. Appl. Phys., 82[12], 6293-6300 (1997).

10) G. Kresse, J. Furthmüller, "Efficiency of ab-initio total energy calculations for metals and semiconductors using a plane-wave basis set," Comput. Mater. Sci., 6[1], 15-50 (1996).

11) G. Kresse, J. Furthmüller, "Efficient iterative schemes for $a b$ initio total-energy calculations using a plane-wave basis set," Phys. Rev., B54[16], 11169-11186 (1996).

12) J. P. Perdew, K. Burke, M. Ernzerhof, "Generalized gradient approximation made simple," Phys. Rev. Lett., 77[18], 38653868 (1996).

13) P. E. Blöchl, "Projector augmented-wave method," Phys. Rev., B50[24], 17953-17979 (1994).

14) G. Kresse, D. Joubert, "From ultrasoft pseudopotentials to the projector augmented-wave method," Phys. Rev., B59[3], 17581775 (1999).

15) A. V. Barashev, A. C. Arokiam, "Monte Carlo modelling of $\mathrm{Cu}$ atom diffusion in $\alpha$-Fe via the vacancy mechanism," Philos. Mag. Lett., 86[5], 321-332 (2006).

16) C. Kittel, Introduction to Solid State Physics, 8th Ed., John Wiley \& Sons, New York (2005).

17) A. Vehanen, P. Hautojärvi, J. Johansson, J. Yli-Kauppila, P. Moser, "Vacancies and carbon impurities in $\alpha$-iron: Electron irradiation," Phys. Rev., B25[2], 762-780 (1982). 